In press: Futures and Foresight Science

This manuscript has not been proof corrected.

An open-access online version is available from Wiley publishers at https://onlinelibrary.wiley.com/doi/epdf/10.1002/ffo2.39

Studies Past and Future of the Past and Future: Commentary on Schoemaker 2020

David R. Mandel

Defence Research and Development Canada

Correspondence: drmandel66@gmail.com

Acknowledgement: Funding for this research was provided by Canadian Safety and Security Program project CSSP-2018-TI-2394. 


\section{Future Studies of the Past}

In this thought-provoking essay, Schoemaker argues that studies of the past can inform studies of the future. I agree with this claim, but I found he equivocated on what is being contributed by history. Is it the methodologies that historians use to study the past? Is it the products of historical analysis that might be used as a knowledge base for future projections? Or is it the past itself, which is the subject of history but which is certainly not its equivalent? It would help greatly if this could be clarified (perhaps to some extent in his rejoinder).

For instance, in discussing whether history repeats itself, Schoemaker seems to refer to the past itself, reflecting on whether there are cyclical or quasi-cyclical patterns in nature that can be exploited for better foresight and planning. Yet there is a sense of inevitability to the claim that there are recurrences in human behavior over epochs and that they can be used as a knowledge base for future projections. Would it even be possible to sketch the future without recourse to such knowledge in some form or other? The extraction of such patterns is surely within the historian's purview but historians don't exercise the equivalent of intellectual property rights over the enterprise. Data science, artificial intelligence, behavioral science and other disciplines will capture a larger intellectual market share of pattern detection from archival data, which itself has undergone an exponential increase that remains unabated.

The future of history is itself uncertain. How will future generations value it and to what extent will historical analyses be trusted? How are expert narrative accounts of the past, even today in the era of politicized disinformation and fake 
news generally regarded? Has the breakneck acceleration of Tofflerian information societies transformed (traditionalists might say warped) our temporal and epistemological values? Do we value the future more than the past, and prediction more than explanation, and is the balance of such valuations changing with time? Can we concentrate on the past as well as we had in the bygone past? Will the value of metrification blossom under increasing societal pressures to quantify that which is good, leaving the narrative arts an arcane profession? The uncertain answers to these and other pressing questions will shape the methods by which future generations interpret and extract lessons from the past. Historical methods, taken together as we currently know them, are but one track on an open field.

Past Studies of the Future

Putting these speculative matters aside, I want to focus on the criteria for evaluating the success of studies of the past and future. There is an important temporal asymmetry in that, generally speaking, we know what happened in the past and we don't know what will happen in the future. Historical analyses, therefore, are not graded on accuracy criteria. Nor are they scored on precision, at least not in the sense of narrowing the aperture on an estimate. Rather, historical analyses are scored on the facets of explanatory goodness, such as their coherence, breadth, and parsimony.

Studies of the future can also be scored on explanation-based criteria, but since the future is unknown they could also be graded on accuracy and precision once enough time has passed. On this matter, however, studies of the future fracture into forecast and foresight camps. The former actively embrace grading 
forecast quality in terms of accuracy and have developed a wide range of quantitative scoring methods, most of which are based on some mathematical loss function (Armstrong, 2001; Gneiting \& Rafferty, 2007), many of which can be decomposed into specific skill and non-skill components (Murphy, 1973; Yaniv, Yates, \& Smith, 1991) and some of which can accommodate predictions about ordered sets (Jose, Nau, \& Winkler, 2009). In contrast, foresight studies actively reject the use of accuracy as a basis for grading quality and often lean over backwards to emphasize that their aim is not to forecast.

The asymmetry between these camps in attempts to clarify what their enterprise isn't about is stark. Studies of forecasting don't state with mantric repetition that they are not concerned with "giving shape" to the future, yet it is commonplace for organizational and academic foresight literature to warn that it does not aim to predict. For example, Policy Horizons Canada, a Government of Canada organization that provides strategic foresight, explains, "At Horizons, the objective of foresight is to explore plausible, alternative futures and identify the challenges and opportunities that may emerge.... The objective of foresight is not to predict the future, but to prepare strategies that are robust across a range of plausible futures." Likewise, Schoemaker warns, "The aim of scenarios is not probabilistic forecasting nor to characterize a few uncertainties in terms of their possible outcomes and likelihoods." Instead, he notes that the main purpose is to "develop insightful narratives about possible futures that improve strategic conversations about planning." 
Statements like these can foster false dichotomies. There is no reason why plausible scenarios constructed by futurists could not be assigned probabilities, either to scenarios as a whole or to their parts. Critics might argue that probabilities would be useless in foresight because the analyses project much further into the future than forecasts typically do. One might therefore have to wait a very long time to have outcome information. While in practice, even longer-term forecasts such those made in strategic intelligence (Mandel \& Barnes, 2014, 2018) or forecasting tournaments (Tetlock, Mellers, Rorbaugh, \& Chen, 2014) tend to project less than one year forward, in principle there is no reason why longer-term forecasts could not be tracked for accuracy, and there are many reasons why they should.

Even if accuracy were not tracked, assigning probabilities to scenarios and their parts would enable users to examine the logical coherence of the scenario. To give a toy example, suppose a futurist constructs a threat scenario that has a dozen necessary conditions that are themselves independent probabilistic events. Half of them are estimated to have a $50 \%$ chance of occurrence and the other half are estimated to have a $90 \%$ chance of occurrence. Given these estimates, one can verify if the analyst is coherent, as a coherent judge should estimate the scenario to have roughly a $0.8 \%$ chance of occurring. Such violations of coherence are predictive of inaccuracy in judgment and can be effectively used to weight judges' contributions to a pooled estimate (Fan, Budescu, Mandel, \& Himmelstein, 2019; Karvetski, Olson, Mandel, \& Twardy, 2013). Even is aggregation isn't used, such feedback could give decision-makers an empirical basis to gauge appropriate levels of trustpresumably, one would not want to put much stock in the scenarios of an incoherent 
advisor. Such feedback could also be given to scenario planners so that they could learn how to better internally calibrate their judgments.

In sum, futurists should befriend explicit subjective probabilities and forecasters shouldn't shy away from long-term projections. Societies should invest in tracking forecast accuracy over much longer temporal horizons than currently performed because many important problems require far-reaching foresight. It doesn't take a futurist, let alone a rocket scientist, to understand that the small cost of such ventures would be far outweighed by their benefits, but to get there, escaping history's trend, policymakers will require sufficient foresight. 


\section{References}

Armstrong, J. S. (2001). Evaluating forecasting methods. In J. S. Armstrong (Ed.), Principles of forecasting: A handbook for researchers and practitioners (pp. 443472). Norwell, MA: Kluwer. https://doi.org/10.1007/978-0-306-47630-3_20

Fan, Y., Budescu, D. V., Mandel, D., Himmelstein, M. (2019). Improving accuracy by coherence weighting of direct and ratio probability judgments. Decision Analysis, 16, 197-217.

Gneiting, T., \& Raftery, A. E. (2007). Strictly proper scoring rules, prediction, and estimation. Journal of the American Statistical Association, 102(477), 359-378.

Karvetski, C. W., Olson, K. C., Mandel, D. R., \& Twardy, C. R. (2013). Probabilistic coherence weighting for optimizing expert forecasts. Decision Analysis, 10(4), $305-326$.

Jose, V. R. R., Nau, R. F., \& Winkler, R. L. (2009). Sensitivity to distance and baseline distributions in forecast evaluation. Management Science, 55(4), 582-590.

Mandel, D. R., \& Barnes, A. (2014). Accuracy of forecasts in strategic intelligence. Proceedings of the National Academy of Sciences, 111(30), 1098410989.

Mandel, D. R., \& Barnes, A. (2018). Geopolitical forecasting skill in strategic intelligence. Journal of Behavioral Decision Making, 31(1), 127-137.

Murphy, A. H. (1973). A new vector partition of the probability score. Journal of Applied Meteorology, 12(4), 595-600. 
Policy Horizons Canada. (n.d.). Module 1: Introduction to foresight. Available from https://horizons.gc.ca/en/our-work/learning-materials/foresight-trainingmanual-module-1-introduction-to-foresight/

Tetlock, P. E., Mellers, B. A., Rorbaugh, N., \& Chen, E. (2014). Forecasting tournaments: Tools for increasing transparency and improving the quality of the debate." Current Directions in Psychological Science, 23(4), 290-295.

Yaniv, I., Yates, J. F., \& Smith, J. E. K. (1991) Measures of discrimination skill in probabilistic judgment. Psychological Bulletin, 110(3), 611-617. 